\title{
On Optimal Properties of Special Nonlinear and Semi-infinite Problems Arising in Parametric Optimization
}

\author{
Olga Kostyukova $^{1}$, Tatiana Tchemisova ${ }^{2, *}$, Maria Kurdina ${ }^{1}$ \\ ${ }^{1}$ Institute of Mathematics, National Academy of Sciences of Belarus, Belarus. \\ 2 Department of Mathematics, University of Aveiro, Portugal.
}

(Received: 19 April 2017; Accepted: 11 May 2017)

\begin{abstract}
We consider a special Nonlinear Programming problem depending on integer parameters. For some values of these parameters (the "right" ones), this problem satisfies certain properties used in study of differential properties of optimal solutions in parametric Semi-Infinite Programming. We deduce the conditions guaranteing the existence of the "right" parameters values, and propose an algorithm for their determination. The conditions and the algorithm are essentially based on properties of a related linear-quadratic semi-infinite problem.
\end{abstract}

Keywords Parametric Optimization, Semi-Infinite programming, Nonlinear Programming

AMS 2010 subject classifications 90C25, 90C30, 90C34

DOI: $10.19139 /$ soic.v5i2.303

\section{Parametric NLP problem: statement and motivation}

Suppose that the index sets

$$
I=I_{1} \cup I_{2}, I_{1} \cap I_{2}=\emptyset,\left|I_{1}\right| \leq n, J,|J| \leq n,
$$

matrices, vectors, and numbers

$$
\begin{gathered}
D_{j} \in \mathbb{R}^{p \times p}, A_{j} \in \mathbb{R}^{n \times p}, B_{j} \in \mathbb{R}^{s_{j} \times p}, c_{j} \in \mathbb{R}^{p}, m_{j} \in \mathbb{R}, m_{j}>0, j \in J, \\
D \in \mathbb{R}^{n \times n}, c \in \mathbb{R}^{n}, q_{i} \in \mathbb{R}^{n}, \omega_{i} \in \mathbb{R}, i \in I
\end{gathered}
$$

are given and fixed.

Suppose also that

$$
\begin{gathered}
K(j)=\left\{l \in \mathbb{R}^{p}: B_{j} l \leq 0\right\}, j \in J \\
D=D^{T}, x^{T} D x \geq 0 \quad \forall x \in \mathbb{R}^{n}, D_{j}=D_{j}^{T}, t^{T} D_{j} t \geq 0 \text { for all } t \in K(j), j \in J,
\end{gathered}
$$

and

$$
\text { relations } \sum_{i \in I} q_{i} \eta_{i}=0, \eta_{i} \geq 0, i \in I_{2} \text {, imply the inequality }-\sum_{i \in I} \omega_{i} \eta_{i} \geq 0 \text {. }
$$

\footnotetext{
*Correspondence to: Tatiana Tchemisova (Email: tatiana@ua.pt). Department of Mathematics, University of Aveiro. Campus Universitário Santiago, Aveiro, Portugal.
}

ISSN 2310-5070 (online) ISSN 2311-004X (print)

Copyright (C) 2017 International Academic Press 
For a given set of integers $p_{j} \geq 1, j \in J$, consider the following problem:

$$
\begin{gathered}
\min F(\xi):=\frac{1}{2} x^{T} D x+\sum_{j \in J} \sum_{k=1}^{p_{j}} y_{k j}\left(\frac{1}{2} t_{k j}^{T} D_{j} t_{k j}+c_{j}^{T} t_{k j}\right)-\sum_{i \in I} \omega_{i} y_{i}, \\
\text { s.t. } \mathcal{F}(\xi):=D x+\sum_{j \in J} A_{j} \sum_{k=1}^{p_{j}} y_{k j} t_{k j}+\sum_{i \in I} q_{i} y_{i}+c=0, \\
y_{i} \geq 0, i \in I_{2} ; \sum_{k=1}^{p_{j}} y_{k j}=m_{j}, y_{k j} \geq 0, t_{k j} \in K(j), k=1, \ldots, p_{j} ; j \in J,
\end{gathered}
$$

where

$$
\xi=\xi\left(p_{j}, j \in J\right)=\left(x, t_{k j}, y_{k j}, k=1, \ldots, p_{j}, j \in J ; y_{i}, i \in I\right)
$$

is a vector of decision variables. In what follows, we will denote problem (6) by $P\left(p_{j}, j \in J\right)$.

The origin of problem (6) and the importance of investigation of its properties are discussed in [8].

Notice that fulfillment of the implication (5) is a necessary condition for boundedness from below of the cost function of the problem $P\left(p_{j}, j \in J\right)$. Moreover, due to this implication (see [8]), in problem (6), without loss of generality we may consider that

$$
\operatorname{rank}\left(q_{i}, i \in I_{1}\right)=\left|I_{1}\right| \text {. }
$$

The problem $P\left(p_{j}, j \in J\right)$ is a Nonlinear Programming problem (NLP) in a special form. With fixed values $y_{k j}, k=1, \ldots, p_{j}, j \in J$ (in particular, when $p_{j}=1, j \in J$ ), the problem $P\left(p_{j}, j \in J\right.$ ) becomes a nonconvex Quadratic Programming (QP) problem ([4]), hence it can be considered as a weighted QP problem that incorporates additional nonlinearities.

It is well known that the majority of NLP problems arising in applications have special forms (see, for example $[1,7])$. A detailed study of such problems, taking into consideration their specific structures, permits one to get more strong theoretical results and build efficient numerical methods ([5, 6]). Auxiliary problems in the form $P\left(p_{j}, j \in J\right)$ with data as in (1), (2) arise, for example, in study of parametric SIP problems with finitely representable index sets ([8]). In our subsequent paper dedicated to the parametric SIP, we will show that the differential properties of solutions of the parametric SIP problems can be formulated in terms of solutions of the problem $P\left(p_{j}, j \in J\right)$. Therefore it is important to provide a deep study of this problem and its properties w.r.t. the values of the parameters $p_{j}, j \in J$.

In [8], it is shown that we are interested in the values of parameters $p_{j} \geq 1, j \in J$, for which the corresponding problem $P\left(p_{j}, j \in J\right)$ admits an optimal solution

$$
\xi^{0}=\xi^{0}\left(p_{j}, j \in J\right)=\left(x^{0}, t_{k j}^{0}, y_{k j}^{0}, k=1, \ldots, p_{j}, j \in J ; y_{i}^{0}, i \in I\right)
$$

possessing the following properties.

Property 1: The components $y_{k j}^{0}, k=1, \ldots, p_{j}, j \in J$, of the optimal solution are strictly positive:

$$
y_{k j}^{0}>0, k=1, \ldots, p_{j}, j \in J .
$$

Property 2:

$$
\operatorname{rank}\left(A_{j}\left(t_{k j}^{0}-t_{1 j}^{0}\right), k=2, \ldots, p_{j}, j \in J_{*}, q_{i}, i \in I_{1} \cup I_{2}^{a}\right)=\left|I_{2}^{a}\right|+\sum_{j \in J} p_{j}+\gamma_{*},
$$

where $J_{*}=\left\{j \in J: p_{j} \geq 2\right\}, I_{2}^{a}=\left\{i \in I_{2}: y_{i}^{0}>0\right\}, \gamma_{*}:=\left|I_{1}\right|-|J| . \quad$ Note that $\left|I_{2}^{a}\right|+\sum_{j \in J} p_{j}+\gamma_{*}=\left|I_{2}^{a}\right|+$ $\left|I_{1}\right|+\sum_{j \in J}\left(p_{j}-1\right)$, and it follows from (11) that

$$
\left|I_{2}^{a}\right|+\left|I_{1}\right|+\sum_{j \in J}\left(p_{j}-1\right) \leq n
$$


Property 3: For any $j \in J$, vectors

$$
t_{k j}^{0}, k \in\left\{s \in\left\{1, \ldots, p_{j}\right\}: y_{s j}^{0}>0\right\}
$$

are global optimal solutions in the problem

$$
\min \Psi_{j}(t):=\left(\frac{1}{2} t^{T} D_{j} t+\left(c_{j}-A_{j}^{T} x^{0}\right)^{T} t\right), \text { s.t. } t \in K(j) .
$$

Note that problem (13) is quadratic but not convex.

The aims of this paper are the following:

- to find new conditions that guarantee the existence of the values of the parameters $p_{j} \geq 1, j \in J$, such that the corresponding problem $P\left(p_{j}, j \in J\right)$ possesses the Properties 1) - 3). We will call these values the right ones;

- to formulate an associated SIP problem that is closely connected with the NLP problem $P\left(p_{j}, j \in J\right)$ with the right values of the parameters $p_{j} \geq 1, j \in J$;

- to develop an algorithm for finding such right values of the parameters.

\section{Some additional properties of optimal solutions of the problem $P\left(p_{j}, j \in J\right)$}

In the previous sections, we have formulated the Properties 1) - 3) that are satisfied by the optimal solutions of problem (6) when choosing the right values of parameters $p_{j}, j \in J$. In this section, we present some results connected with the optimality properties of this problem. These results were proved in [8] and will be used in the next sections.

Theorem 1 (Sufficient optimality conditions)

Let vector $\xi^{0}$ in the form (9) be a feasible solution of problem (6) and let the following conditions be fulfilled:

1. $\xi^{0}$ satisfies the relations

$$
q_{i}^{T} x^{0}+\omega_{i}=0, i \in I_{1} ; q_{i}^{T} x^{0}+\omega_{i} \leq 0, y_{i}^{0}\left(q_{i}^{T} x^{0}+\omega_{i}\right)=0, i \in I_{2} ;
$$

2. for $j \in J$, vectors (12) are global optimal solutions of problem (13).

Then the vector $\xi^{0}$ is a global optimal solution of problem (6).

\section{Lemma 1}

Suppose that problem (6) has an optimal solution $\xi^{0}$ in the form (9) such that $y_{k_{0} j_{0}}^{0}=0$ for some $1 \leq k_{0} \leq p_{j_{0}}$, $j_{0} \in J$. Then

$$
\operatorname{val}\left(P\left(\bar{p}_{j}, j \in J\right)\right)=\operatorname{val}\left(P\left(p_{j}, j \in J\right)\right),
$$

where $\bar{p}_{j}=p_{j}, j \in J \backslash\left\{j_{0}\right\}, \bar{p}_{j_{0}}=p_{j_{0}}-1$.

Here and in what follows, $\operatorname{val}(P)$ denotes the optimal value of the cost function in an optimization problem $P$.

Lemma 2

Let integers $\bar{p}_{j}, p_{j}, j \in J$, satisfy the inequalities $\bar{p}_{j} \geq p_{j}, j \in J$. Then

$$
\operatorname{val}\left(P\left(\bar{p}_{j}, j \in J\right)\right) \leq \operatorname{val}\left(P\left(p_{j}, j \in J\right)\right) .
$$

Lemma 3

Let a feasible solution (9) of problem $P\left(p_{j}, j \in J\right)$ satisfy conditions (14) and Property 3 ). Then for all integers $\bar{p}_{j} \geq p_{j}, j \in J$, the equality

$$
\operatorname{val}\left(P\left(\bar{p}_{j}, j \in J\right)\right)=\operatorname{val}\left(P\left(p_{j}, j \in J\right)\right)
$$

takes the place. 
The following lemma was proved in our previous paper (see Lemma 4.6 in [8]). Since some steps of the proof of this lemma will be used in a new algorithm for finding the "right" parameters' values, we present here the proof.

Lemma 4

Suppose that the problem $P\left(p_{j}, j \in J\right)$ has an optimal solution satisfying Property 3$)$. Then there exist integers $1 \leq \bar{p}_{j} \leq p_{j}, j \in J$, such that problem $P\left(\bar{p}_{j}, j \in J\right)$ has an optimal solution satisfying Properties 1)- 3).

Proof. Suppose that the problem $P\left(p_{j}, j \in J\right)$ has an optimal solution satisfying Property 3$)$. If this solution does not satisfy Property 1 ), then applying Lemma 1 , one can easily find numbers $\tilde{p}_{j} \leq p_{j}, j \in J$, such that the problem $P\left(\tilde{p}_{j}, j \in J\right)$ has an optimal solution

$$
\xi^{0}\left(\tilde{p}_{j}, j \in J\right)=\left(x^{0}, t_{k j}^{0}, y_{k j}^{0}, k=1, \ldots, \tilde{p}_{j}, j \in J ; y_{i}^{0}, i \in I\right)
$$

satisfying Property 1):

$$
y_{k j}^{0}>0, k=1, \ldots, \tilde{p}_{j}, j \in J
$$

Evidently, Property 3$)$ is also satisfied by $\xi^{0}\left(\tilde{p}_{j}, j \in J\right)$.

Denote $J_{*}:=\left\{j \in J: \tilde{p}_{j} \geq 2\right\}$. Suppose that Property 2$)$ is not satisfied for $\xi^{0}\left(\tilde{p}_{j}, j \in J\right)$, i.e.

$$
m\left(\xi^{0}\left(\tilde{p}_{j}, j \in J\right)\right)<\left|I_{2}^{a}\right|+\sum_{j \in J} \tilde{p}_{j}+\gamma_{*},
$$

where $m\left(\xi^{0}\left(\tilde{p}_{j}, j \in J\right)\right):=\operatorname{rank}\left(A_{j}\left(t_{k j}^{0}-t_{1 j}^{0}\right), k=2, \ldots, \tilde{p}_{j}, j \in J_{*}, q_{i}, i \in I_{1} \cup I_{2}^{a}\right)$. Hence vectors

$$
\left(\begin{array}{c}
A_{j} t_{k j}^{0} \\
e_{j}
\end{array}\right), k=1, \ldots, \tilde{p}_{j}, j \in J_{*}, \text { and }\left(\begin{array}{c}
q_{i} \\
\mathbf{0}
\end{array}\right), i \in I_{1} \cup I_{2}^{a},
$$

where

$$
e_{j}=\left(e_{i j}, i \in J\right)^{T}, e_{i j}=0 \text { if } i \neq j, \quad e_{i j}=1 \text { if } i=j, i \in J, j \in J, \mathbf{0}=(0,0, \ldots, 0)^{T} \in \mathbb{R}^{|J|},
$$

are linearly dependent. Consequently, there exists a vector

$$
\left(\eta_{k j}, k=1, \ldots, \tilde{p}_{j}, j \in J_{*}, \eta_{i}, i \in I_{1} \cup I_{2}^{a}\right) \neq 0
$$

such that

$$
\sum_{j \in J_{*}} \sum_{k=1}^{\tilde{p}_{j}} \eta_{k j} A_{j} t_{k j}^{0}+\sum_{i \in I_{1} \cup I_{2}^{a}} q_{i} \eta_{i}=0, \sum_{k=1}^{\tilde{p}_{j}} \eta_{k j}=0, j \in J_{*} .
$$

Set $\eta_{1 j}:=0, j \in J \backslash J_{*}$, and

$$
\begin{array}{r}
\lambda_{k j}=\infty \text { if } \eta_{k j} \geq 0, \lambda_{k j}=-y_{k j}^{0} / \eta_{k j} \text { if } \eta_{k j}<0, k=1, \ldots, \tilde{p}_{j}, j \in J ; \\
\lambda_{i}=\infty \text { if } \eta_{i} \geq 0, \lambda_{i}=-y_{i}^{0} / \eta_{i} \text { if } \eta_{i}<0, i \in I_{2}^{a} .
\end{array}
$$

Then, evidently,

$$
\lambda:=\min \left\{\lambda_{k j}, k=1, \ldots, \tilde{p}_{j}, j \in J ; \lambda_{i}, i \in I_{2}^{a}\right\}>0 .
$$

Consider the numbers

$$
\bar{y}_{k j}^{0}:=y_{k j}^{0}+\lambda \eta_{k j}, k=1, \ldots, \tilde{p}_{j}, j \in J ; \quad \bar{y}_{i}^{0}:=y_{i}^{0}+\lambda \eta_{i}, i \in I_{1} \cup I_{2}^{a}, \bar{y}_{i}^{0}:=y_{i}^{0}, i \in I \backslash\left(I_{1} \cup I_{2}^{a}\right) .
$$

By construction, we have

$$
\sum_{k=1}^{\tilde{p}_{j}} \bar{y}_{k j}^{0}=m_{j}, \bar{y}_{k j}^{0} \geq 0, k=1, \ldots, \tilde{p}_{j}, j \in J ; \quad \bar{y}_{i}^{0} \geq 0, i \in I_{2} .
$$


Due to Property 3) and taking into account inequalities (16), it is easy to show that for all $j \in J$, the following relations take place:

$$
-\sum_{k=1}^{\tilde{p}_{j}} y_{k j}^{0}\left(\frac{1}{2} t_{k j}^{0 T} D_{j} t_{k j}^{0}+c_{j}^{T} t_{k j}^{0}\right)=\sum_{k=1}^{\tilde{p}_{j}} y_{k j}^{0}\left(x^{0 T} A_{j} t_{k j}^{0}-\lambda(j)\right)=\sum_{k=1}^{\tilde{p}_{j}} y_{k j}^{0} x^{0 T} A_{j} t_{k j}^{0}-\lambda(j) m_{j} .
$$

Hence

$$
\begin{gathered}
-\sum_{k=1}^{\tilde{p}_{j}} y_{k j}^{0}\left(\frac{1}{2} t_{k j}^{0 T} D_{j} t_{k j}^{0}+c_{j}^{T} t_{k j}^{0}\right)=\sum_{k=1}^{\tilde{p}_{j}} y_{k j}^{0} x^{0 T} A_{j} t_{k j}^{0}-\lambda(j) m_{j}, \\
-\sum_{k=1}^{\tilde{p}_{j}} \bar{y}_{k j}^{0}\left(\frac{1}{2} t_{k j}^{0 T} D_{j} t_{k j}^{0}+c_{j}^{T} t_{k j}^{0}\right)=\sum_{k=1}^{\tilde{p}_{j}} \bar{y}_{k j}^{0} x^{0 T} A_{j} t_{k j}^{0}-\lambda(j) m_{j} \\
=-\sum_{k=1}^{\tilde{p}_{j}} y_{k j}^{0}\left(\frac{1}{2} t_{k j}^{0 T} D_{j} t_{k j}^{0}+c_{j}^{T} t_{k j}^{0}\right)+\sum_{k=1}^{\tilde{p}_{j}} \eta_{k j} x^{0 T} A_{j} t_{k j}^{0} .
\end{gathered}
$$

Taking into account the last relations and (18), it is easy to show one can conclude that

$$
F\left(\xi^{0}\left(\tilde{p}_{j}, j \in J\right)\right)=F\left(\bar{\xi}^{0}\left(\tilde{p}_{j}, j \in J\right)\right),
$$

where $\xi^{0}\left(\tilde{p}_{j}, j \in J\right)$ is defined in (15) and

$$
\bar{\xi}^{0}\left(\tilde{p}_{j}, j \in J\right):=\left(x^{0}, t_{k j}^{0}, \bar{y}_{k j}^{0}, k=1, \ldots, \tilde{p}_{j}, j \in J ; \bar{y}_{i}^{0}, i \in I\right) .
$$

From the considerations above, it follows that $\bar{\xi}^{0}\left(\tilde{p}_{j}, j \in J\right)$ is an optimal solution of the problem $P\left(\tilde{p}_{j}, j \in J\right)$.

Notice that, by construction,

$$
\min \left\{\bar{y}_{k j}^{0}, k=1, \ldots, \tilde{p}_{j}, j \in J ; \bar{y}_{i}^{0}, i \in I_{2}^{a}\right\}=0 .
$$

Following Lemma 1, let us find numbers $\overline{\tilde{p}}_{j} \leq \tilde{p}_{j}, j \in J$, such that the vector

$$
\bar{\xi}^{0}\left(\overline{\tilde{p}}_{j}, j \in J\right):=\left(x^{0}, t_{k j}^{0}, \bar{y}_{k j}^{0}, k=1, \ldots, \overline{\tilde{p}}_{j}, j \in J ; \bar{y}_{i}^{0}, i \in I\right)
$$

is optimal for the problem $P\left(\overline{\tilde{p}}_{j}, j \in J\right)$ and $\bar{y}_{k j}^{0}>0, k=1, \ldots, \overline{\tilde{p}}_{j}, j \in J$.

It is easy to check that

$$
m\left(\bar{\xi}^{0}\left(\overline{\tilde{p}}_{j}, j \in J\right)\right)=m\left(\xi^{0}\left(\tilde{p}_{j}, j \in J\right)\right), \quad\left|I_{2}^{a}\right|+\sum_{j \in J} \tilde{p}_{j}>\left|\bar{I}_{2}^{a}\right|+\sum_{j \in J} \overline{\tilde{p}}_{j}
$$

where

$$
\begin{aligned}
m\left(\bar{\xi}^{0}\left(\overline{\tilde{p}}_{j}, j \in J\right)\right) & :=\operatorname{rank}\left(A_{j}\left(t_{k j}^{0}-t_{1 j}^{0}\right), k=2, \ldots, \overline{\tilde{p}}_{j}, j \in \bar{J}_{*}, q_{i}, i \in I_{1} \cup \bar{I}_{2}^{a}\right), \\
\bar{I}_{2}^{a} & :=\left\{i \in I_{2}: \bar{y}_{i}^{0}>0\right\}, \bar{J}_{*}:=\left\{j \in J: \overline{\tilde{p}}_{j} \geq 2\right\} .
\end{aligned}
$$

It follows from (17) and (21) that in a finite number of iterations, we will find numbers $\bar{p}_{j} \leq p_{j}, j \in J$, and an optimal solution of the problem $P\left(\bar{p}_{j}, j \in J\right)$ such that Properties 1)-3) are satisfied for this solution. The lemma is proved. 


\section{A related SIP problem}

In this section, we consider a SIP problem of a special form possessing some special properties. We will show how an optimal solution of this problem permits to find parameters $p_{j}, j \in J$, such that the optimal solution of the corresponding NLP problem $P\left(p_{j}, j \in J\right)$ satisfies Properties 1)-3). The results of this section are useful for different applications and will be used in the future work dedicated to parametric SIP.

Using the source data (1)-(4), let us formulate the following SIP problem:

$$
\begin{array}{cl}
\min _{x \in \mathbb{R}^{n}, \rho_{j} \in \mathbb{R}, j \in J} & \frac{1}{2} x^{T} D x+c^{T} x-\sum_{j \in J} m_{j} \rho_{j} \\
\text { s.t. } & q_{i}^{T} x+\omega_{i}=0, i \in I_{1} ; q_{i}^{T} x+\omega_{i} \leq 0, i \in I_{2} ; \\
& -\frac{1}{2} t_{j}^{T} D_{j} t_{j}-\left(c_{j}-A_{j}^{T} x\right)^{T} t_{j}+\rho_{j} \leq 0, \quad \forall t_{j} \in K(j), j \in J .
\end{array}
$$

Let us make some observations concerning this problem.

- Problem (22) is not parametric.

- In problem (22), the constraints are linear and the cost function is linear-quadratic w.r.t. decision variables $x \in \mathbb{R}^{n}, \rho_{j} \in \mathbb{R}, j \in J$. Hence it is evident that this problem is convex, and any of its local optimal solution is a global one.

- If problem (22) is consistent, then the (infinite) constraints $-\frac{1}{2} t_{j}^{T} D_{j} t_{j}-\left(c_{j}-A_{j}^{T} x\right)^{T} t_{j}+\rho_{j} \leq 0, \forall t_{j} \in$ $K(j), j \in J$, satisfy the Slater condition.

- Since $t_{j}=0 \in K(j)$ then it is evident that $\rho_{j} \leq 0, j \in J$.

- For any feasible solution $\left(x, \rho_{j}, j \in J\right)$ of problem (22) the following inequalities are fulfilled:

$$
\left(c_{j}^{T}-x^{T} A_{j}\right) \tau_{j} \geq 0 \forall \tau_{j} \in \Delta K(j):=\left\{\tau \in K(j): \tau^{T} D_{j} \tau=0\right\}, j \in J .
$$

Theorem 2

Suppose that the convex SIP problem (22) has an optimal solution $\left(x^{0} \in \mathbb{R}^{n}, \rho_{j}^{0} \in \mathbb{R}, j \in J\right)$ such that inequalities (23) are fulfilled in the strong form:

$$
\left(c_{j}^{T}-x^{0^{T}} A_{j}\right) \tau_{j}>0 \forall \tau_{j} \in \Delta K(j), j \in J .
$$

Then there exist numbers $p_{j} \geq 1, j \in J, \sum_{j \in J} p_{j} \leq n$, such that the NLP problem $P\left(p_{j}, j \in J\right)$ has an optimal solution satisfying Properties 1)-3).

Proof. It follows from optimality of $\left(x^{0} \in \mathbb{R}^{n}, \rho_{j}^{0} \in \mathbb{R}, j \in J\right)$ in the SIP problem (22) that

$$
\rho_{j}^{0}=\rho_{j}\left(x^{0}\right), \rho_{j}(x):=\min _{t_{j} \in K(j)}\left(\frac{1}{2} t_{j}^{T} D_{j} t_{j}+\left(c_{j}-A_{j}^{T} x\right)^{T} t_{j}\right), j \in J .
$$

Hence all the active index sets

$$
K_{a}(j):=\left\{t_{j} \in K(j): \frac{1}{2} t_{j}^{T} D_{j} t_{j}+\left(c_{j}-A_{j}^{T} x^{0}\right)^{T} t_{j}=\rho_{j}^{0}\right\}, j \in J,
$$

are nonempty. Taking into account the Slater condition, relations (24), and Theorem 1 from [9], it is easy to show that there exist vectors

$$
t_{k j}^{0} \in K_{a}(j), k=1, \ldots, \bar{p}_{j}, j \in J ; \text { where } 1 \leq \bar{p}_{j}, j \in J, \sum_{j \in J} \bar{p}_{j} \leq n,
$$

and numbers $y_{k j}^{0}, k=1, \ldots, \bar{p}_{j}, j \in J ; y_{i}^{0}, i \in I$, such that the following conditions take place:

$$
\begin{gathered}
D x^{0}+\sum_{j \in J} A_{j} \sum_{k=1}^{\bar{p}_{j}} y_{k j}^{0} t_{k j}^{0}+\sum_{i \in I} q_{i} y_{i}^{0}+c=0, \\
y_{i}^{0} \geq 0, y_{i}^{0}\left(q_{i}^{T} x^{0}+\omega_{i}\right)=0, i \in I_{2} ; \sum_{k=1}^{\bar{p}_{j}} y_{k j}^{0}=m_{j}, y_{k j}^{0} \geq 0, k=1, \ldots, \bar{p}_{j} ; j \in J .
\end{gathered}
$$


It follows from (22), (25) and (26) that the vector $\xi^{*}=\left(x^{0}, t_{k j}^{0}, y_{k j}^{0}, k=1, \ldots, \bar{p}_{j}, j \in J ; y_{i}^{0}, i \in I\right)$ is a feasible solution of the problem $P\left(\bar{p}_{j}, j \in J\right)$ and satisfies Property 3$)$. Moreover for $\xi^{*}$, the sufficient optimality conditions formulated in Theorem 1 are satisfied.

Therefore we have shown that the problem $P\left(\bar{p}_{j}, j \in J\right)$ has an optimal solution satisfying Property 3$)$. Applying Lemma 4, we complete the proof of the theorem.

The following theorem states sufficient optimality conditions for a given feasible solution of problem (22).

\section{Theorem 3}

Let $\left(x^{0}, \rho_{j}^{0}, j \in J\right)$ be a feasible solution in the convex SIP problem (22). Suppose that there exist vectors (25), and numbers $y_{k j}^{0}, k=1, \ldots, \bar{p}_{j}, j \in J ; y_{i}^{0}, i \in I$, such that the conditions (26) take place. Then the vector $\left(x^{0}, \rho_{j}^{0}, j \in J\right)$ is an optimal solution of problem (22).

Proof. From the statement of the theorem, it follows that the vector $\left(x^{0}, \rho_{j}^{0}, j \in J\right)$ is an optimal solution of the following convex QP problem:

$$
\begin{array}{cl}
\min _{x \in \mathbb{R}^{n}, \rho_{j} \in \mathbb{R}, j \in J} & \frac{1}{2} x^{T} D x+c^{T} x-\sum_{j \in J} m_{j} \rho_{j} \\
\text { s.t. } & q_{i}^{T} x+\omega_{i}=0, i \in I_{1} ; q_{i}^{T} x+\omega_{i} \leq 0, i \in I_{2} ; \\
& -\frac{1}{2} t_{k j}^{0 T} D_{j} t_{k j}^{0}-\left(c_{j}-A_{j}^{T} x\right)^{T} t_{k j}^{0}+\rho_{j} \leq 0, \quad k=1, \ldots, \bar{p}_{j} ; j \in J .
\end{array}
$$

Evidently, the feasible set of problem (22) is contained in the feasible set of problem (27). Hence, if a feasible solution $\left(x^{0}, \rho_{j}^{0}, j \in J\right)$ of problem (22) is optimal in problem (27), then it will be optimal in problem (22). The theorem is proved.

Finally, we will show that the existence of an optimal solution in the convex SIP problem (22) is necessary for the existence of an optimal solution of problem $P\left(p_{j}, j \in J\right)$ possessing Properties 1)-3).

\section{Theorem 4}

Let problem $P\left(p_{j}, j \in J\right)$ has an optimal solution satisfying Properties 1)-3). Then problem (22) has an optimal solution.

Proof. Let problem $P\left(p_{j}, j \in J\right)$ has an optimal solution satisfying Properties 1)-3). Then one can show that problem $P\left(p_{j}, j \in J\right)$ has a solution

$$
\xi^{0}=\left(x^{0}, t_{k j}^{0}, y_{k j}^{0}, k=1, \ldots, p_{j}, j \in J ; y_{i}^{0}, i \in I\right)
$$

satisfying conditions 1 . and 2. of Theorem 1 . Hence, the vector $\left(x^{0}, \rho_{j}^{0}=\rho_{j}\left(x^{0}\right), j \in J\right)$ is a feasible solution of problem (22) and satisfies conditions (26) with $\bar{p}_{j}=p_{j}, j \in J$. According to Theorem 3, vector $\left(x^{0}, \rho_{j}^{0}=\right.$ $\left.\rho_{j}\left(x^{0}\right), j \in J\right)$ is an optimal solution of problem (22) that proves the theorem.

It is evident that the implication

$$
\Delta x \in \Delta X \Rightarrow c^{T} \Delta x \geq 0
$$

with

$$
\begin{gathered}
\Delta X=\left\{\Delta x \in \mathbb{R}^{n}: q_{i}^{T} \Delta x=0, i \in I_{1} ; q_{i}^{T} \Delta x \leq 0, i \in I_{2} ; D \Delta x=0 ;\right. \\
\left.\exists \mu^{*}(j)=\mu^{*}(\Delta x, j) \geq 0 \text { such that } \Delta x^{T} A_{j}=\mu^{* T}(j) B_{j}, j \in J\right\},
\end{gathered}
$$

is a necessary condition for boundedness from below of the cost function in problem (22). 
Moreover, let us show (see Lemma 5) that the implication (28) is a necessary condition for feasibility of problem $P\left(p_{j}, j \in J\right)$.

Lemma 5

Suppose that the set of feasible solutions in problem $P\left(p_{j}, j \in J\right)$ is nonempty, then implication (28) takes place.

Proof.

It is evident that for all values of integers $p_{j} \geq 1, j \in J$, the set of feasible solutions in problem $P\left(p_{j}, j \in J\right)$ is nonempty if and only if the set of feasible solutions in problem $P\left(p_{j}=1, j \in J\right)$ is nonempty.

Suppose that the set of feasible solutions in problem $P\left(p_{j}=1, j \in J\right)$ is nonempty, i. e. there exists a vector

$$
\left(x^{*}, t_{j}^{*}, j \in J ; y_{i}^{*}, i \in I\right)
$$

such that

$$
\begin{gathered}
D x^{*}+c+\sum_{j \in J} m_{j} A_{j} t_{j}^{*}+\sum_{i \in I} q_{i} y_{i}^{*}=0, \\
y_{i}^{*} \geq 0, i \in I_{2} ; t_{j}^{*} \in K(j), j \in J,
\end{gathered}
$$

but implication (28) does not take place, i.e. $\exists \Delta x^{*} \in \Delta X$ such that $c^{T} \Delta x^{*}<0$. Let us multiply (30) by $\Delta x^{* T}$ taking into account the last relations. As a result we obtain

$$
0=\Delta x^{* T} c+\sum_{j \in J} m_{j} \Delta x^{* T} A_{j} t_{j}^{*}+\sum_{i \in I_{2}} \Delta x^{* T} q_{i} y_{i}^{*} \leq \Delta x^{* T} c<0 .
$$

The obtained contradiction proves that implication (28) takes place. The lemma is proved.

One can prove the following theorem that gives sufficient optimality conditions for the existence of an optimal solution in problem (22).

\section{Theorem 5}

Suppose that the following conditions are satisfied:

A) the implication (5) is true and there exists

$$
\bar{x} \in\left\{x \in \mathbb{R}^{n}: q_{i}^{T} x+\omega_{i}=0, i \in I_{1} ; q_{i}^{T} x+\omega_{i} \leq 0, i \in I_{2}\right\}
$$

such that

$$
\left(c_{j}^{T}-\bar{x}^{T} A_{j}\right) \tau_{j} \geq 0 \forall \tau_{j} \in \Delta K(j):=\left\{\tau \in K(j): \tau^{T} D_{j} \tau=0\right\}, j \in J ;
$$

B) given the set $\Delta X$ defined in (29), either $\Delta X \backslash\{0\}$ is empty or the following implication takes place:

$$
\Delta x \in \Delta X \backslash\{0\} \Rightarrow c^{T} \Delta x>0 .
$$

Then SIP problem (22) has an optimal solution.

\section{Determination of the "right" values of the parameters $p_{j}, j \in J$, in the problem $P\left(p_{j}, j \in J\right)$}

In this section we use the results of the section 3 to develop an algorithm that finds the values of parameters $p_{j}, j \in J$, for which problem $P\left(p_{j}, j \in J\right)$ satisfies Properties 1) -3$)$. Notice that this algorithm differs from the one presented in [8] and works under less strict assumptions.

Algorithm 1

Step 1. Using the data given in (1)-(4), construct a SIP problem in the form (22) and solve it. If problem (22) has no optimal solutions, STOP: according to Theorem 4 there do not exist parameters $p_{j}, j \in J$, such that problem $P\left(p_{j}, j \in J\right)$ has an optimal solution satisfying Properties 1)-3). Otherwise go to Step 2. 
Step 2. Let $\left(x^{0}, \rho_{j}^{0}, j \in J\right)$ be an optimal solution of the SIP problem (22).

Suppose that relations (24) take place.

Find vectors $t_{k j}^{0} \in K_{a}(j), k=1, \ldots, \bar{p}_{j}, j \in J$, (see (25)) and numbers $y_{k j}^{0}, k=1, \ldots, \bar{p}_{j}, j \in J ; y_{i}^{0}, i \in I$, such that conditions (26) take place. (According to Theorem 2, such vectors and numbers exist and the vector $\xi^{*}=\left(x^{0}, t_{k j}^{0}, y_{k j}^{0}, k=1, \ldots, \bar{p}_{j}, j \in J ; y_{i}^{0}, i \in I\right)$ is a feasible solution of the problem $P\left(\bar{p}_{j}, j \in J\right)$ satisfying Property 3).)

Set $s:=1, t_{k j}^{0}(s):=t_{k j}^{0}, y_{k j}^{0}(s):=y_{k j}^{0}, k=1, \ldots, \bar{p}_{j}, p_{j}(s):=\bar{p}_{j}, j \in J ; y_{i}^{0}(s):=y_{i}^{0}, i \in I$. Go to Step 3 .

Step 3. Let

$$
\xi(s):=\left(x^{0} ; t_{k j}^{0}(s), y_{k j}^{0}(s), k=1, \ldots, p_{j}(s), j \in J ; y_{i}^{0}(s), i \in I\right)
$$

be an optimal solution of problem $P\left(p_{j}(s), j \in J\right)$ satisfying Property 3$)$.

If, additionally, $\xi(s)$ satisfies Property $1: y_{k j}^{0}(s)>0$ for all $k=1, \ldots, p_{j}(s), j \in J$, then go to Step 4.

Suppose that for some $j^{*} \in J$ there exists $k^{*}:=k\left(j^{*}\right)$ such that $y_{k^{*} j^{*}}^{0}(s)=0$.

Then set:

$$
\begin{gathered}
p_{j}(s+1):=p_{j}(s) \text { for all } j \in J \backslash\left\{j^{*}\right\} \text { and } p_{j^{*}}(s+1):=p_{j^{*}}(s)-1 ; \\
t_{k j}^{0}(s+1):=t_{k j}^{0}(s), y_{k j}^{0}(s+1):=y_{k j}^{0}(s), k=1, \ldots, p_{j}(s+1), j \in J \backslash\left\{j^{*}\right\} ; \\
t_{k j^{*}}^{0}(s+1):=t_{k j^{*}}^{0}(s), y_{k j_{*}}^{0}(s+1):=y_{k j}^{0}(s), k=1, \ldots, k^{*}-1, \\
t_{k j^{*}}^{0}(s+1):=t_{k+1 j^{*}}^{0}(s), y_{k j_{*}}^{0}(s+1):=y_{k+1 j^{*}}^{0}(s), k=k^{*}, \ldots, p_{j^{*}}(s+1), \\
y_{i}^{0}(s+1):=y_{i}^{0}(s), i \in I .
\end{gathered}
$$

Set $s:=s+1$ and repeat Step 3 .

Step 4. At this step we have a set of parameters $p_{j}(s), j \in J$, for which the optimal solution $\xi(s)$ of the problem $P\left(p_{j}(s), j \in J\right)$ in the form (33) satisfies Properties 1) and 3).

If $\xi(s)$ satisfies Property 2), then STOP; the set of parameters $p_{j}:=p_{j}(s), j \in J$, is the desired one.

Otherwise, following the algorithmic proof of Lemma 4, set

$$
J^{*}:=\left\{j \in J: p_{j}(s) \geq 2\right\}, \quad I_{2}^{a}:=\left\{i \in I_{2}: y_{i}^{0}(s)>0\right\} .
$$

Solve the system

$$
\sum_{j \in J_{*}} \sum_{k=1}^{p_{j}(s)} \eta_{k j} A_{j} t_{k j}^{0}+\sum_{i \in I_{1} \cup I_{2}^{a}} q_{i} \eta_{i}=0, \sum_{k=1}^{p_{j}(s)} \eta_{k j}=0, j \in J_{*} .
$$

Let $\left(\eta_{k j}, k=1, \ldots, p_{j}(s), j \in J_{*} ; \eta_{i}, i \in I_{1} \cup I_{2}^{a}\right)$ be its nontrivial solution (that exists since Property 2 ) is not satisfied). Set $\eta_{1 j}:=0, j \in J \backslash J_{*}$. Using the found values $\eta_{k j}, k=1, \ldots, p_{j}(s), j \in J ; \eta_{i}, i \in I_{1} \cup I_{2}^{a}$, calculate the number $\lambda>0$ by formulas (19) and (20) where $\tilde{p}_{j}, j \in J$, are replaced by $p_{j}(s), j \in J$.

Set $p_{j}(s+1):=p_{j}(s), j \in J$, and

$$
\xi(s+1):=\left(x^{0} ; t_{k j}^{0}(s), y_{k j}^{0}(s+1), k=1, \ldots, p_{j}(s+1), j \in J ; y_{i}^{0}(s+1), i \in I\right),
$$

where

$$
\begin{gathered}
y_{k j}^{0}(s+1)=y_{k j}^{0}(s)+\lambda \eta_{k j}, k=1, \ldots, p_{j}(s+1), j \in J \\
y_{i}^{0}(s+1)=y_{i}^{0}(s)+\lambda \eta_{i}, i \in I_{1} \cup I_{2}^{a}, y_{i}^{0}(s+1)=y_{i}^{0}(s), i \in I \backslash\left(I_{1} \cup I_{2}^{a}\right) .
\end{gathered}
$$

Notice that according to Lemma 4, it holds: $F\left(\xi^{0}(s)=F\left(\xi^{0}(s+1)\right.\right.$, and

$$
\min \left\{y_{k j}^{0}(s+1), k=1, \ldots, p_{j}(s+1), j \in J ; y_{i}^{0}(s+1), i \in I_{2}^{a}\right\}=0 .
$$

Set $s:=s+1$ and repeat Step 3 of the algorithm.

The algorithm is described. It follows from Lemma 4 that the algorithm is finite. 


\section{Conclusions}

In this paper, we have considered a class of parametric NLP problems $P\left(p_{j}, j \in J\right)$ in the special form (6). We are especially interested in the values of parameters $p_{j} \geq 1, j \in J$, for which problem $P\left(p_{j}, j \in J\right)$ has an optimal solution possessing Properties 1) - 3).

Using the specific structure of problems $P\left(p_{j}, j \in J\right)$ and in-depth analysis of their properties we have obtained the following results.

- We have formulated the first order sufficient optimality conditions and studied in details how the change of the parameters in a problem $P\left(p_{j}, j \in J\right)$ affects the optimal value of its cost function.

- Given data (1) and (2), we have formulated a convex SIP problem in a special form (22) and showed how this SIP problem is connected with the original NLP problem $P\left(p_{j}, j \in J\right)$. In particular,

- we proved that if there exists an optimal solution of the SIP problem such that relations (24) take place, then there exist parameters' values $p_{j}, j \in J$, such that the optimal solution of the problem $P\left(p_{j}, j \in J\right)$ satisfies the Properties 1)-3);

- we proved that if an optimal solution of the problem $P\left(p_{j}, j \in J\right)$ satisfies the Properties 1)-3) then the SIP problem (22) admits an optimal solutions;

- Finally, we have constructed an algorithm which in a finite number of iterations either finds the values of the parameters for which the corresponding problem $P\left(p_{j}, j \in J\right)$ has optimal solutions satisfying the Properties 1) - 3) or states that such parameters do not exist.

The results of the paper are interesting since they can be used in study of the parametric SIP problems with finitely representable index sets. Our future paper will be dedicated to such a study and will actively use the results obtained here.

\section{Acknowledgement}

This work was partially supported by Belarusian State Scientific Program "Convergence" and Portuguese funds through CIDMA - Center for Research and Development in Mathematics and Applications, and FCT - Portuguese Foundation for Science and Technology, within the project UID/MAT/04106/2013.

\section{REFERENCES}

1. A. Ben-Tal, and M. Teboulle, Hidden convexity in some nonconvex quadratically constrained quadratic programming, Mathematical Programming, no. 72, pp. 51-63, 1996.

2. J. F. Bonnans, and A. Shapiro, Perturbation analysis of optimization problems, Springer-Verlag, New-York, 2000.

3. B. C. Eaves, On Quadratic Programming, Management Science, vol. 17, no. 11, Theory Series, pp. 698-711, 1971.

4. C. A. Floudas, and V. Visweswaran, Quadratic programming, in Handbook of Global Optimization. Kluwer Academic Publishers, Dordrecht, 1995.

5. P. E. Gill, W. Murray, and M.H.Wright, Practical optimization, Academic Press Inc., London, 1981.

6. R. Horst, P.M. Pardalos, and N.V. Thoai, Introduction to Global Optimization: non-convex optimization and its applications, Kluwer Academic Publishers, Dordrech, 2000.

7. S. Kim, and M. Koima, Exact solutions of some nonconvex quadratic optimization problems via SDP and SOCP relaxations, Computational Optimization and Applications, no 26, pp.143154, 2003.

8. O. I. Kostyukova, T. V. Tchemisova, and M. A.Kurdina, A study of one class of NLP problems arising in parametric Semi-Infinite Programming, Optimization Methods and Software, in press, 2016.

9. V.L. Levin, Application of E. Helly's theorem to convex programming, problems of best approximation and related questions, Math. USSR Sbornik, vol. 8, no. 2, pp. 235-247, 1969. 